\title{
Green function solution of generalised boundary value problems
}

\author{
Vanik E. Mkrtchian* $\nsim$ and Carsten Henkel ${ }^{\sharp}$ \\ * Institute for Physical Research, Armenian Academy of Sciences, Ashtarak 0203, \\ Republic of Armenia \\ \# University of Potsdam, Institute of Physics and Astronomy, Karl-Liebknecht-Str. \\ 24/25, 14476 Potsdam, Germany
}

\begin{abstract}
We construct an expression for the Green function of a differential operator satisfying nonlocal, homogeneous boundary conditions starting from the fundamental solution of the differential operator. This also provides the solution to the boundary value problem of an inhomogeneous partial differential equation with inhomogeneous, nonlocal, and linear boundary conditions. The construction generally applies for all types of linear partial differential equations and linear boundary conditions.

PACS numbers:
\end{abstract}

Submitted to: J. Phys. A: Math. Gen.

\section{Introduction}

There are a number of examples where the use of Feynman's path integral approach has provided simplifications, new results or a better understanding of already known facts. Because it has a completely different starting point compared to canonical quantum field theory, the functional integral may identify unknown aspects of a problem and increase understanding by adding complementary considerations. One item on this list is our earlier work on a simple one-dimensional boundary value problem involving the Helmholtz operator with Robin boundary conditions at the endpoints of an interval [1]. We solved the problem using Feynmans' path integral and introduced auxiliary fields to take into account the boundary conditions. We came to an expression for the Green function of the problem that is very natural to generalize to a broader class of differential operators, dimensions, and boundary conditions.

$\S$ vem@daad-alumni.de 
It is the purpose of this paper to present this generalization and a few of its consequences. We construct a Green function for a differential operator with homogeneous boundary conditions and use it to solve in a simple, but general way a linear inhomogeneous equation involving that differential operator and constrained by inhomogeneous linear boundary conditions. The latter are taken in the form of an boundary integral and thus may be called non-local.

Throughout the paper, we use the traditional field theory language (the functional integral representation is avoided). The issues of existence and uniqueness of the solutions are left for future work, assuming for the moment that we deal with wellposed problems in Hadamard's sense [2]. As potential applications we have in mind the electrodynamic response of interfaces with spatially dispersive materials that play a key role in dispersion forces [3] and radiative heat transfer across sub-micron vacuum gaps [4]. In that context, the Green function provides a compact way to compute the elements of the energy-momentum tensor of the field within a bounded region, for example between two plates. It contains (multiply) reflected waves that arise from the boundary conditions considered on the plates. We show here how to connect the reflection amplitudes in a simple and general way to the imposed boundary conditions.

\section{Problem formulation}

We consider a differential operator $\mathfrak{L}$ defined on $\mathbb{R}^{n}$, a domain $\Omega \subset \mathbb{R}^{n}$ and want to construct a Green function $G_{x^{\prime}}: \Omega \rightarrow \mathbb{C}$ that solves

$$
\mathfrak{L} G_{x^{\prime}}=\delta_{x^{\prime}}
$$

for a source point $x^{\prime}$ in the interior of $\Omega . \delta_{x^{\prime}}$ is the unit-mass Dirac distribution supported at $x^{\prime}\left[5 \mid\right.$. We shall often write $G_{x^{\prime}}(x)=G\left(x, x^{\prime}\right)$.

The Green function is constrained by a finite set of homogeneous, nonlocal boundary conditions $(j=1, \ldots m)$

$$
\forall \bar{x} \in \partial \Omega: \quad \int_{\partial \Omega} \mathrm{d} S\left(\bar{x}_{1}\right) b_{j}\left(\bar{x}, \bar{x}_{1}\right) G\left(\bar{x}_{1}, x^{\prime}\right)=0
$$

In this expression, $\mathrm{d} S\left(\bar{x}_{1}\right)$ is the Lebesgue measure on the boundary domain $\partial \Omega \ni \bar{x}_{1}$, and the $b_{j}: \partial \Omega \times \partial \Omega \rightarrow \mathbb{C}$ are well-behaved integral kernels. Generally speaking, each kernel $b_{j}$ is defined on its own sub-domain $\partial \Omega_{j}$ of the boundary surface $\partial \Omega=$ $\partial \Omega_{1} \cup \partial \Omega_{2} \cup \ldots \cup \partial \Omega_{m}$. The surface integral (2) can then be restricted to the subdomain $\partial \Omega_{j}$.

In other words, the set of kernels $\left\{b_{j} \mid j=1, \ldots m\right\}$ defines a linear map (integral

operator) $\hat{B}$ from the space of complex functions defined on the boundary domain $\partial \Omega$ to "spinor-valued" functions $\hat{\varphi}: \partial \Omega \rightarrow \mathbb{C}^{m}$. The restriction $\bar{G}_{x^{\prime}}: \partial \Omega \rightarrow \mathbb{C}, \bar{G}_{x^{\prime}}(\bar{x})=G_{x^{\prime}}(\bar{x})$ of the Green function $G_{x^{\prime}}$ is in the kernel of this map, $\hat{B} \bar{G}_{x^{\prime}}=\hat{0}$. In the following, we use the word "boundary function" for a function defined on $\partial \Omega$. 
We also need the adjoint $\hat{B}^{\dagger}$ of the map $\hat{B}$ : it maps spinor-valued boundary functions $\hat{\varphi}$ to scalar functions and is defined by the boundary integral

$$
\left(\hat{B}^{\dagger} \hat{\varphi}\right)(\bar{x})=\sum_{j} \int_{\partial \Omega} \mathrm{d} S\left(\bar{x}_{1}\right) b_{j}^{*}\left(\bar{x}_{1}, \bar{x}\right) \varphi_{j}\left(\bar{x}_{1}\right)
$$

where $b_{j}^{*}\left(\bar{x}_{1}, \bar{x}\right)=\left[b_{j}\left(\bar{x}_{1}, \bar{x}\right)\right]^{*}$ is the complex conjugate.

We assume that the fundamental solution $E$ of the differential operator $\mathfrak{L}[6]$, i.e., the solution to

$$
\mathfrak{L} E_{x^{\prime}}=\delta_{x^{\prime}}
$$

in $\mathbb{R}^{n}$ is known: $E_{x^{\prime}}(x)=E\left(x, x^{\prime}\right)$. Note that $E_{x^{\prime}}$ is actually a member of a class of solutions because it is not constrained by boundary conditions.

Finally, we consider apart from the original boundary value problem (1, 2) also its adjoint version: the corresponding Green function is denoted $G_{x^{\prime}}^{a}$ and solves

$$
\mathfrak{L}^{a} G_{x^{\prime}}^{a}=\delta_{x^{\prime}}
$$

in the domain $\Omega$. In terms of the natural sesquilinear form on $\Omega$, this means

$$
\left\langle\mathfrak{L} u, G_{x^{\prime}}^{a}\right\rangle=\int_{\Omega} \mathrm{d} V(x)[(\mathfrak{L} u)(x)]^{*} G^{a}\left(x, x^{\prime}\right)=u^{*}\left(x^{\prime}\right)
$$

for all $x^{\prime} \in \Omega$ and functions $u: \Omega \rightarrow \mathbb{C}$ in the domain of $\mathfrak{L}$. Here, the volume integral involves the familiar Lebesgue measure $\mathrm{d} V(x)$ in the domain $\Omega$. The boundary condition for the adjoint problem is

$$
\hat{B}^{a} \bar{G}_{x^{\prime}}^{a}=0
$$

where $\hat{B}^{a}$ has also $m$ components with kernels $b_{j}^{a}\left(\bar{x}, \bar{x}_{1}\right)$, as in $(2)$. We shall write $E_{x^{\prime}}^{a}$ for the fundamental solution to (5) in $\mathbb{R}^{n}$.

It is a well-known result of the theory of linear differential equations (see for example Refs. [6], [7]) that the Green function $G\left(x, x^{\prime}\right)$ and its adjoint counterpart $G^{a}\left(x, x^{\prime}\right)$ are linked by

$$
G^{a}\left(x, x^{\prime}\right)=G^{*}\left(x^{\prime}, x\right)
$$

\section{Boundary integral representation of the Green function}

We claim that the Green function $G_{x^{\prime}}$ to the problem (1, 2) can be expressed via the fundamental solution $E$ in the following way

$$
\forall x \in \Omega: \quad G\left(x, x^{\prime}\right)=E\left(x, x^{\prime}\right)-\int_{\partial \Omega} \mathrm{d} S(\bar{x}) E(x, \bar{x}) J_{x^{\prime}}^{(1)}(\bar{x})
$$

where the integral runs over the boundary $\partial \Omega$ and involves the boundary function $J_{x^{\prime}}^{(1)}: \partial \Omega \rightarrow \mathbb{C}$. The latter is constructed by applying a sequence of linear maps

$$
J_{x^{\prime}}^{(1)}=\hat{B}_{1}^{\dagger} \hat{\mathbf{g}}^{-1} \hat{B} \bar{E}_{x^{\prime}}
$$


to the restriction $\bar{E}_{x^{\prime}}$ of the fundamental solution $E_{x^{\prime}}$ to the boundary $\partial \Omega$.

The key element in the construction $(9)$ is the inverse $\hat{\mathbf{g}}^{-1}$ of the $m \times m$ matrix operator $\hat{\mathbf{g}}$, a linear map between spinor-valued functions on the boundary. The matrix elements of $\hat{\mathrm{g}}$ are given by the double boundary integrals $(1 \leq i, j \leq m)$

$$
g_{i j}\left(\bar{x}, \bar{x}^{\prime}\right)=\int_{\partial \Omega \times \partial \Omega} \mathrm{d} S\left(\bar{x}_{1}\right) \mathrm{d} S\left(\bar{x}_{2}\right) b_{i}\left(\bar{x}, \bar{x}_{1}\right) E\left(\bar{x}_{1}, \bar{x}_{2}\right) b_{1 j}^{*}\left(\bar{x}^{\prime}, \bar{x}_{2}\right)
$$

where the kernels $b_{1 j}$ are the components of the boundary operator $\hat{B}_{1}$.

Proof. We apply the differential operator $\mathfrak{L}$ to both sides of $9 a$. The fundamental solution in the first term gives the Dirac distribution $\delta_{x^{\prime}}$. In the second term, we pull $\mathfrak{L}$ under the boundary integral and get zero because $x^{\prime}$ is in the interior of $\Omega$, while $\bar{x}_{1}$ is not.

The boundary condition (2) follows from the construction (9c) of the matrix operator $\hat{\mathbf{g}}$ : the application of $\hat{B}$ to the second term in $(9 a)$ generates under the integral the operator product $\hat{B} \bar{E} \hat{B}_{1}^{\dagger}=\hat{\mathrm{g}}$ applied to the spinor function $\hat{\varphi}=\hat{\mathbf{g}}^{-1} \hat{B} \bar{E}_{x^{\prime}}$. The boundary integral thus reduces to $\hat{B} \bar{E}_{x^{\prime}}$ which cancels the first term.

Obviously the solution $G_{x^{\prime}}$ exists if the kernel $(9 c)$ defines an invertible map $\hat{\mathrm{g}}[6]$. We assume that this holds if we deal with a well-posed problem [2].

The above demonstration is still lacking an explicit expression for the operator $\hat{B}_{1}$. To find it, we first write down a similar solution to the adjoint problem (5, 7):

$$
\begin{aligned}
G^{a}\left(x, x^{\prime}\right) & =E^{a}\left(x, x^{\prime}\right)-\int_{\partial \Omega} \mathrm{d} S(\bar{x}) E^{a}(x, \bar{x}) J_{x^{\prime}}^{(2)}(\bar{x}) \\
J_{x^{\prime}}^{(2)}(\bar{x}) & =\hat{B}_{2}^{\dagger}(\hat{\mathbf{h}})^{-1} \hat{B}^{a} \bar{E}_{x^{\prime}}^{a} \\
h_{i j}\left(\bar{x}, \bar{x}^{\prime}\right) & =\int_{\partial \Omega \times \partial \Omega} \mathrm{d} S\left(\bar{x}_{1}\right) \mathrm{d} S\left(\bar{x}_{2}\right) b_{i}^{a}\left(\bar{x}, \bar{x}_{1}\right) E^{a}\left(\bar{x}_{1}, \bar{x}_{2}\right) b_{2 j}^{*}\left(\bar{x}^{\prime}, \bar{x}_{2}\right)
\end{aligned}
$$

where $\hat{B}_{2}$ is another boundary operator. The proof that this solves the adjoint problem follows the same lines as above. (It is useful to note the concise form of $(10 c)$ : $\hat{\mathbf{h}}=\hat{B}^{a} \bar{E} \hat{B}_{2}^{\dagger}$.)

Because of the ambiguity of the unbounded fundamental solutions, we may always assume that they satisfy the identity (8), i.e.

$$
E^{a}\left(x, x^{\prime}\right)=E^{*}\left(x^{\prime}, x\right)
$$

By requiring that the matrix elements $h_{i j}[\mathrm{Eq} \cdot(10 c)]$ and $g_{j i}^{*}[\mathrm{Eq} \cdot(9 c)]$ coincide, we find component-wise the identification

$$
\hat{B}_{1}=\hat{B}^{a}, \quad \hat{B}_{2}=\hat{B}
$$


We insert this Ansatz into $G^{*}\left(x^{\prime}, x\right)$ from the complex conjugate of $9 a$ and switch the boundary integral over $E^{*}\left(x^{\prime}, \bar{x}\right) J_{x}^{(1) *}(\bar{x})$ written there with the application of $\hat{B} \bar{E}_{x}$ in $(9 b)$. It is then easy to check that one gets the identity

$$
\hat{B}_{2}^{\dagger}(\hat{\mathbf{h}})^{-1} \hat{B}^{a}=\hat{B}^{\dagger} \hat{\mathbf{g}}^{-1 \dagger} \hat{B}_{1}=\hat{B}_{1}^{\dagger *} \hat{\mathbf{g}}^{-1 *} \hat{B}^{*}
$$

The last step in 13 is based on the observation that in the position representation, the two-variable kernel of this expression is a scalar function. Therefore, one may take the formal transpose of the operator product. This yields (8) between $G$ and $G^{a}$.

When the solution $(12)$ is inserted into $(9)$, we find the explicit expressions

$$
\begin{aligned}
G\left(x, x^{\prime}\right) & =E\left(x, x^{\prime}\right)-\int_{\partial \Omega} \mathrm{d} S(\bar{x}) E(x, \bar{x}) J_{x^{\prime}}^{(1)}(\bar{x}) \\
J_{x^{\prime}}^{(1)} & =\hat{B}^{a \dagger} \hat{\mathbf{g}}^{-1} \hat{B} \bar{E}_{x^{\prime}} \\
g_{i j}\left(\bar{x}, \bar{x}^{\prime}\right) & =\int_{\partial \Omega \times \partial \Omega} \mathrm{d} S\left(\bar{x}_{1}\right) \mathrm{d} S\left(\bar{x}_{2}\right) b_{i}\left(\bar{x}, \bar{x}_{1}\right) E\left(\bar{x}_{1}, \bar{x}_{2}\right) b_{j}^{a *}\left(\bar{x}^{\prime}, \bar{x}_{2}\right)
\end{aligned}
$$

Remark. It follows from this that the Green function also obeys

$$
G\left(x, x^{\prime}\right) \overleftarrow{\mathfrak{L}}_{x^{\prime}}=\delta\left(x-x^{\prime}\right)
$$

with differential operator and boundary condition acting from the right:

$$
\int_{\partial \Omega} \mathrm{d} S\left(\bar{x}^{\prime}\right) G\left(x, \bar{x}^{\prime}\right) \hat{B}_{j}^{a \dagger}\left(\bar{x}^{\prime}, \bar{x}\right)=0
$$

Example. As a simple application of the solution (14) to the boundary value problem (1), let us construct the Green function of a self-adjoint Dirichlet problem, i.e.

$$
\begin{aligned}
& \mathfrak{L}_{x} G_{D}\left(x, x^{\prime}\right)=\delta\left(x-x^{\prime}\right), \quad x, x^{\prime} \in \Omega \\
& G_{D}\left(\bar{x}, x^{\prime}\right)=G_{D}^{a}\left(\bar{x}, x^{\prime}\right)=0, \quad \bar{x} \in \partial \Omega
\end{aligned}
$$

Let $E\left(x, x^{\prime}\right)$ be the fundamental solution to 117$)$, then we find from the definition $14 c$ that the matrix operator $\hat{\mathrm{g}}$ reduces to a scalar kernel

$$
g\left(\bar{x}, \bar{x}^{\prime}\right)=E\left(\bar{x}, \bar{x}^{\prime}\right)
$$

The solution (14) reads in this case

$$
\begin{aligned}
G\left(x, x^{\prime}\right)=E & \left(x, x^{\prime}\right) \\
& -\int_{\partial \Omega \times \partial \Omega} \mathrm{d} S(\bar{x}) \mathrm{d} S\left(\bar{x}^{\prime}\right) E(x, \bar{x}) g^{-1}\left(\bar{x}, \bar{x}^{\prime}\right) E\left(\bar{x}^{\prime}, x^{\prime}\right)
\end{aligned}
$$

where $g^{-1}$ is the inverse of the boundary integral operator with kernel 19$)$ defined on the manifold $\partial \Omega$ :

$$
\begin{aligned}
& \int_{\partial \Omega} \mathrm{d} S\left(\bar{x}_{1}\right) E\left(\bar{x}, \bar{x}_{1}\right) g^{-1}\left(\bar{x}_{1}, \bar{x}^{\prime}\right)= \\
& \int_{\partial \Omega} \mathrm{d} S\left(\bar{x}_{1}\right) g^{-1}\left(\bar{x}, \bar{x}_{1}\right) E\left(\bar{x}_{1}, \bar{x}^{\prime}\right)=\delta\left(\bar{x}, \bar{x}^{\prime}\right)
\end{aligned}
$$




\section{Boundary value problem}

The above construction provides an integral representation that solves a boundary value problem for the inhomogeneous equation

$$
\mathfrak{L} u=f
$$

with a smooth function $f: \Omega \rightarrow \mathbb{C}$. The boundary conditions can be cast in a fairly general form as a set of $m$ inhomogeneous integral equations for the restriction $\bar{u}$ of $u$ to the boundary:

$$
\hat{B} \bar{u}=\hat{\Phi}
$$

Here, the given "spinor" $\hat{\Phi}$ has the boundary functions $\Phi_{j}: \partial \Omega \rightarrow \mathbb{C}$ as its components. Explicitly, Eq. (23) reads

$$
\forall \bar{x} \in \partial \Omega: \quad \int_{\partial \Omega} \mathrm{d} S\left(\bar{x}_{1}\right) b_{j}\left(\bar{x}, \bar{x}_{1}\right) u\left(\bar{x}_{1}\right)=\Phi_{j}(\bar{x}) \quad(j=1, \ldots m)
$$

by direct analogy to (2).

We claim that when the boundary value problem defined by 22 is well-posed à la Hadamard, then its solution $u$ is given by the expression

$$
u(x)=\int_{\Omega} \mathrm{d} V\left(x_{1}\right) G\left(x, x_{1}\right) f\left(x_{1}\right)+\int_{\partial \Omega} \mathrm{d} S(\bar{x}) E(x, \bar{x}) J_{\hat{\Phi}}(\bar{x})
$$

Under the boundary integral (second line), the complex-valued boundary function $J_{\hat{\Phi}}$ depends linearly on the $\Phi_{j}$ 's:

$$
J_{\hat{\Phi}}=\hat{B}^{a \dagger} \hat{\mathbf{g}}^{-1} \hat{\Phi}
$$

in close analogy to $14 a$.

Proof. When acting on both sides of $(25 a)$ with $\mathfrak{L}$, we find $f$ because of (1). Applying the operator $\hat{B}$ on the boundary restriction $\bar{u}$, we find $\hat{\Phi}$ because of (2) and the definition $(14 c)$ of the boundary operators $\hat{\mathbf{g}}$ and $\hat{\mathbf{g}}^{-1}$.

\section{Examples}

\subsection{Local boundary conditions}

For a Cauchy initial value problem or for boundary value problems with Dirichlet, Neumann or Robin boundary conditions, the operator $\hat{B}$ in $(3)$ acts in a local way:

$$
\left(\hat{B} \bar{G}_{x^{\prime}}\right)(\bar{x})=\hat{B}(\bar{x}) \bar{G}_{x^{\prime}}(\bar{x})=0
$$


The expression (14) for the Green function then reads

$$
\begin{aligned}
& G\left(x, x^{\prime}\right)= E\left(x, x^{\prime}\right) \\
&-\int_{\partial \Omega \times \partial \Omega} \mathrm{d} S\left(\bar{x}_{1}\right) \mathrm{d} S\left(\bar{x}_{2}\right) E\left(x, \bar{x}_{1}\right) \hat{K}\left(\bar{x}_{1}, \bar{x}_{2}\right) \bar{E}\left(\bar{x}_{2}, x^{\prime}\right) \\
& \hat{K}\left(\bar{x}_{1}, \bar{x}_{2}\right)=\hat{B}^{a \dagger}\left(\bar{x}_{1}\right) \hat{\mathbf{g}}^{-1}\left(\bar{x}_{1}, \bar{x}_{2}\right) \hat{B}\left(\bar{x}_{2}\right)
\end{aligned}
$$

Here, the local operators appear to the left and right of the $m \times m$ matrix kernel $\hat{\mathrm{g}}^{-1}\left(\bar{x}, \bar{x}^{\prime}\right)$. The latter is the inverse of the matrix operator $\hat{\mathrm{g}}$ defined on the boundary manifold $\partial \Omega$ in terms of the matrix elements

$$
\hat{g}_{i j}\left(\bar{x}, \bar{x}^{\prime}\right)=\hat{b}_{i}(\bar{x}) E\left(\bar{x}, \bar{x}^{\prime}\right) \hat{b}_{j}^{a *}\left(\bar{x}^{\prime}\right)
$$

The solution of the boundary value problem (22) with local boundary conditions

$$
\forall \bar{x} \in \partial \Omega: \quad \hat{B}(\bar{x}) u(\bar{x})=\hat{\Phi}(\bar{x})
$$

is

$$
\begin{aligned}
& u(x)=\int_{\Omega} \mathrm{d} V\left(x_{1}\right) G\left(x, x_{1}\right) f\left(x_{1}\right)+\int_{\partial \Omega} \mathrm{d} S(\bar{x}) E(x, \bar{x}) \hat{B}^{a \dagger}(\bar{x}) \hat{J}_{\hat{\Phi}}^{\prime}(\bar{x}) \\
& \hat{J}_{\hat{\Phi}}^{\prime} \quad=\hat{\mathbf{g}}^{-1} \hat{\Phi}
\end{aligned}
$$

\subsection{One-dimensional boundary value problem}

In the case of ordinary differential equations, we deal with a one-dimensional problem defined on the interval $\Omega=(a, b)$. In this case, the boundary manifold is the set of two endpoints $\partial \Omega=\{a, b\}$. We then have local boundary conditions that may be expanded in the form

$$
a_{j 0} G_{x^{\prime}}(a)+a_{j 1} \partial_{x} G_{x^{\prime}}(a)+b_{j 0} G_{x^{\prime}}(b)+b_{j 1} \partial_{x} G_{x^{\prime}}(b)=0
$$

where $a_{j 0}, a_{j 1}, b_{j 0}$ and $b_{j 1}(j=1, \ldots m)$ are constants whose values encode whether this linear combination corresponds to the Dirichlet, Neumann oder Robin type. Obviously, the object $\hat{\mathrm{g}}$ in $(27 c)$ reduces in this case to a numerical matrix rather than a matrixvalued integral operator.

The action of the operator $\hat{b}_{j}$ from the left on the fundamental solution $E$ in (27) is in this context to be understood as

$$
\begin{aligned}
& \hat{b}_{j}(\bar{x}) E\left(\bar{x}, x^{\prime}\right) \rightarrow \\
& \quad a_{j 0} E\left(a, x^{\prime}\right)+a_{j 1} \partial_{x} E\left(a, x^{\prime}\right)+b_{j 0} E\left(b, x^{\prime}\right)+b_{j 1} \partial_{x} E\left(b, x^{\prime}\right)
\end{aligned}
$$

where in $\partial_{x} E$, the differentiation is with respect to the first argument of $E$. The action of the operator $\hat{b}_{j}^{a *}$ from the right is defined as

$$
\begin{aligned}
& E\left(x, \bar{x}^{\prime}\right) \hat{b}_{j}^{a *}\left(\bar{x}^{\prime}\right) \rightarrow \\
& \quad a_{j 0}^{a *} E(x, a)+a_{j 1}^{a *} \partial_{x^{\prime}} E(x, a)+b_{j 0}^{a *} E(x, b)+b_{j 1}^{a *} \partial_{x^{\prime}} E(x, b)
\end{aligned}
$$

where $a_{j 0}^{a}, a_{j 1}^{a}, b_{j 0}^{a}$ and $b_{j 1}^{a}$ are constants appearing in the adjoint boundary conditions, and the derivative $\partial_{x^{\prime}} E$ is with respect to the second argument of $E$. 


\section{Concluding remarks}

In a previous paper [1], we constructed the electromagnetic Green function in a bounded domain subject to (nonlocal) boundary conditions at the interface between spatially dispersive media. We have shown here that this result can be generalized to give a boundary integral representation for the Green function related to a broad class of linear partial differential equations with linear homogeneous and nonlocal boundary conditions. This Green function provides the solution to a boundary value problem for linear, inhomogeneous partial differential equations subject to nonlocal, inhomogeneous conditions on the boundary manifold.

\section{Appendix A.}

In this Appendix, we use the block matrix inversion formula [8]

$$
\begin{aligned}
\left(\begin{array}{ll}
\mathbf{A} & \mathbf{B} \\
\mathbf{C} & \mathbf{D}
\end{array}\right)^{-1} & =\left[\begin{array}{cc}
\mathbf{A}^{-1}+\mathbf{A}^{-1} \mathbf{B R C A}^{-1}, & -\mathbf{A}^{-1} \mathbf{B R} \\
-\mathbf{R C A}^{-1}, & \mathbf{R}
\end{array}\right] \\
\mathbf{R} & =\left(\mathbf{D}-\mathbf{C A}^{-1} \mathbf{B}\right)^{-1}
\end{aligned}
$$

to find a simple expression for the inverse of the matrix operator $\hat{\mathbf{g}}$ [Eq. $(14 c)]$ and of the Green function [Eq.(14a)]. The resulting expression for the Green function is

$$
G\left(x, x^{\prime}\right)=E\left(x, x^{\prime}\right)-\sum_{j=1}^{m} G_{j}\left(x, x^{\prime}\right)
$$

with the recursive construction

$$
\begin{aligned}
G_{j}\left(x, x^{\prime}\right) & =\int_{\partial \Omega^{\times 4}} G^{(j-1)}\left(x, \bar{y}_{1}\right) \hat{b}_{j}^{a *}\left(\bar{y}_{2}, \bar{y}_{1}\right) g_{j}^{-1}\left(\bar{y}_{2}, \bar{y}_{2}^{\prime}\right) \hat{b}_{j}\left(\bar{y}_{2}^{\prime}, \bar{y}_{1}^{\prime}\right) \\
& \times G^{(j-1)}\left(\bar{y}_{1}^{\prime}, x^{\prime}\right) \\
g_{j}\left(\bar{x}, \bar{x}^{\prime}\right) & =\int_{\partial \Omega^{\times 2}} \hat{b}_{j}(\bar{x}, \bar{y}) G^{(j-1)}\left(\bar{y}, \bar{y}^{\prime}\right) \hat{b}_{j}^{a *}\left(\bar{x}^{\prime}, \bar{y}^{\prime}\right) \\
G^{(j-1)} & =E-G_{1}-\ldots-G_{j-1} \\
G^{(0)} & \equiv E
\end{aligned}
$$

In these expressions, an integral over $\partial \Omega$ with respect to every doubly appearing variable $\bar{y}_{1}, \ldots \bar{y}_{2}^{\prime}$ has to be performed. The necessary condition is that the kernels $g_{j}$ can be inverted on the boundary manifold $\partial \Omega$ :

$$
\begin{aligned}
& \int_{\partial \Omega} \mathrm{d} S\left(\bar{x}_{1}\right) g_{j}\left(\bar{x}, \bar{x}_{1}\right) g_{j}^{-1}\left(\bar{x}_{1}, \bar{x}^{\prime}\right)= \\
& \int_{\partial \Omega} \mathrm{d} S\left(\bar{x}_{1}\right) g_{j}^{-1}\left(\bar{x}, \bar{x}_{1}\right) g_{j}\left(\bar{x}_{1}, \bar{x}^{\prime}\right)=\delta\left(\bar{x}, \bar{x}^{\prime}\right)
\end{aligned}
$$


Green function solution of generalised boundary value problems

Proof. To verify this result, we act in the following way: we start from $m=1$ where (14) reads

$$
\begin{aligned}
G\left(x, x^{\prime}\right) & =E\left(x, x^{\prime}\right)-G_{1}\left(x, x^{\prime}\right) \\
G_{1}\left(x, x^{\prime}\right) & =\int_{\partial \Omega^{\times 4}} E\left(x, \bar{x}_{1}\right) \hat{b}_{1}^{a *}\left(\bar{x}_{2}, \bar{x}_{1}\right) g_{11}^{-1}\left(\bar{x}_{2}, \bar{x}_{2}^{\prime}\right) \hat{b}_{1}\left(\bar{x}_{2}^{\prime}, \bar{x}_{1}^{\prime}\right) E\left(\bar{x}_{1}^{\prime}, x^{\prime}\right) \\
g_{11}\left(\bar{x}, \bar{x}^{\prime}\right) & =\int_{\partial \Omega^{\times 2}} \hat{b}_{1}\left(\bar{x}, \bar{x}_{1}\right) E\left(\bar{x}_{1}, \bar{x}_{1}^{\prime}\right) \hat{b}_{1}^{a *}\left(\bar{x}^{\prime}, \bar{x}_{1}^{\prime}\right)
\end{aligned}
$$

We see that the expressions A.4 coincide with $\mathrm{A} .2$ for $m=1$.

Introducing the shorthand based on $14 c$

$$
g_{i j} \equiv g_{i j}\left(\bar{x}, \bar{x}^{\prime}\right)=\int_{\partial \Omega^{\times 2}} \hat{b}_{i}\left(\bar{x}, \bar{x}_{1}\right) E\left(\bar{x}_{1}, \bar{x}_{1}^{\prime}\right) \hat{b}_{j}^{a *}\left(\bar{x}^{\prime}, \bar{x}_{1}^{\prime}\right)
$$

we find the inverse of the matrix operator $\hat{\mathbf{g}}$ for $m=2$ using the formula A.1

$$
\left(\begin{array}{ll}
g_{11} & g_{12} \\
g_{21} & g_{22}
\end{array}\right)^{-1}=\left[\begin{array}{cc}
g_{11}^{-1}+g_{11}^{-1} g_{12} R g_{21} g_{11}^{-1}, & -g_{11}^{-1} g_{12} R \\
-R g_{21} g_{11}^{-1}, & R
\end{array}\right]
$$

where $R=\left(g_{22}-g_{21} g_{11}^{-1} g_{12}\right)^{-1}=g_{2}^{-1}$ [see Eq. A.2c)]. And then, inserting (A.6) into expression $14 a$ for the Green function, we find

$$
G=E-G_{1}-G_{2}
$$

which is coincident with $\mathrm{A} .2 a$ ) for $m=2$ and where $G_{2}$ is defined by $\mathrm{A} .2 b$ for $j=2$.

In the case of $m=3$ we deal with $3 \times 3 \hat{g}$ matrix which we invert using A.1 taking

$$
\begin{array}{ll}
\mathbf{A} \rightarrow\left(\begin{array}{ll}
g_{11} & g_{12} \\
g_{21} & g_{22}
\end{array}\right) & \mathbf{B} \rightarrow\left(\begin{array}{l}
g_{13} \\
g_{23}
\end{array}\right) \\
\mathbf{C} \rightarrow\left(\begin{array}{ll}
g_{31} & g_{32}
\end{array}\right) & \mathbf{D} \rightarrow g_{33}
\end{array}
$$

After some algebra, we arrive again at A.2 for $m=3$.

Remark. And finally, let us consider the case of local boundary conditions where the operator $\hat{b}_{i}$ acts in the domain $\partial \Omega_{i}$ of the boundary manifold $\partial \Omega$. Then, two integrals drop out in $\mathrm{A} .2 b, \mathrm{~A} .2 c$, and we find

$$
\begin{aligned}
G_{j}\left(x, x^{\prime}\right) & =\int_{\partial \Omega_{j}^{\times 2}} G^{(j-1)}\left(x, \bar{y}_{1}\right) \hat{b}_{j}^{a *}\left(\bar{y}_{1}\right) g_{j}^{-1}\left(\bar{y}_{1}, \bar{y}_{1}^{\prime}\right) \hat{b}_{j}\left(\bar{y}_{1}^{\prime}\right) G^{(j-1)}\left(\bar{y}_{1}^{\prime}, x^{\prime}\right) \\
g_{j}\left(\bar{x}, \bar{x}^{\prime}\right) & =\hat{b}_{j}(\bar{x}) G^{(j-1)}\left(\bar{x}, \bar{x}^{\prime}\right) \hat{b}_{j}^{a *}\left(\bar{x}^{\prime}\right)
\end{aligned}
$$

\section{Acknowledgments}

We thank Nikolai Tarkhanov for helpful remarks. 
[1] V.E. Mkrtchian and C. Henkel, Ann. Phys. (Berlin) 526 (2014) 87, arXiv:quant-ph/1307.0682.

[2] J. Hadamard, Sur les problèmes aux dérivées partielles et leur signification physique, Princeton University Bulletin 13 (1902) 49-52.

[3] D. A. R. Dalvit, P.W. Milonni, D. Roberts, and F. da Rosa, Casimir Physics, Lecture Notes in Physics vol. 834, Springer, Berlin 2011.

[4] I. A. Dorofeyev and E. A. Vinogradov, Phys. Rep. 504 (2011) 75-143.

[5] R. P. Kanwal, Generalized Functions: Theory and Technique, Academic Press, New York 1983.

[6] I. Stakgold and M. Holst, Green's Functions and Boundary Value Problems, Wiley \& Sons 2011.

[7] M. D. Greenberg, Applications of Green's Functions in Science and Engineering, Dover 2015.

[8] F. Zhang, Matrix Theory, Basic Results and Techniques, Springer, New York 2011. 